\title{
Modules, networks and systems medicine for understanding disease and aiding diagnosis
}

\author{
Mika Gustafsson ${ }^{1 \dagger}$, Colm E Nestor ${ }^{1 \dagger}$, Huan Zhang ${ }^{1 \dagger}$, Albert-László Barabási ${ }^{2}$, Sergio Baranzini ${ }^{3}$, Sören Brunak ${ }^{4,5}$, \\ Kian Fan Chung ${ }^{6}$, Howard J Federoff ${ }^{7}$, Anne-Claude Gavin ${ }^{8}$, Richard R Meehan ${ }^{9}$, Paola Picotti ${ }^{10}$, Miguel Àngel Pujana ${ }^{11}$, \\ Nikolaus Rajewsky ${ }^{12}$, Kenneth GC Smith ${ }^{13,14}$, Peter J Sterk ${ }^{15}$, Pablo Villoslada ${ }^{16}$ and Mikael Benson ${ }^{1 *}$
}

\begin{abstract}
Many common diseases, such as asthma, diabetes or obesity, involve altered interactions between thousands of genes. High-throughput techniques (omics) allow identification of such genes and their products, but functional understanding is a formidable challenge. Network-based analyses of omics data have identified modules of disease-associated genes that have been used to obtain both a systems level and a molecular understanding of disease mechanisms. For example, in allergy a module was used to find a novel candidate gene that was validated by functional and clinical studies. Such analyses play important roles in systems medicine. This is an emerging discipline that aims to gain a translational understanding of the complex mechanisms underlying common diseases. In this review, we will explain and provide examples of how network-based analyses of omics data, in combination with functional and clinical studies, are aiding our understanding of disease, as well as helping to prioritize diagnostic markers or therapeutic candidate genes. Such analyses involve significant problems and limitations, which will be discussed. We also highlight the steps needed for clinical implementation.
\end{abstract}

\section{The complexity of common disease}

Despite impressive advances during the past century, modern health care is faced with enormous challenges. One problem is that currently available drugs show highly variable clinical efficacy, which results not only in suffering, but also contributes to increasing costs. The annual cost of ineffective drugs in the US alone is

\footnotetext{
* Correspondence: mikael.benson@liu.se

${ }^{\dagger}$ Equal contributors

'Centre for Individualized Medicine, Department of Pediatrics, Faculty of Medicine, 58185 Linköping, Sweden

Full list of author information is available at the end of the article
}

estimated at US $\$ 350$ billion [1]. Variable efficacy also adds to the huge costs associated with drug discovery, development and clinical trials (on average US\$1 billion per drug), which further impacts the financing of health care. These problems reflect the complexity of common diseases, which can involve altered interactions between thousands of genes. Because of the large number of genes and their interconnection, it is very difficult to gain functional understanding of disease mechanisms by detailed studies of individual genes.

This problem of complexity is compounded by disease heterogeneity: patients with similar clinical manifestations may have different underlying disease mechanisms. Asthma is an example of such a disease; it can be caused by infection, allergens or other environmental factors, which give rise to different inflammatory responses (Figure 1). Variations in response may underlie the observation that between 10 and $20 \%$ of patients do not respond to one of the most common asthma drugs, corticosteroids [2]. This variation, however, can potentially be exploited to find novel drugs for nonresponders in asthma, allergy and other diseases, as well as to identify patients that require such drugs [3].

Despite the success of single diagnostic markers, there is a pressing need for multiple markers. Single markers are already being used in the clinic to predict disease or personalize treatment and examples include BRCA genotyping in breast cancer, CCR5 mutation status in HIV infection and newborn screening for metabolic defects [4]. Recently, optimization of the anticoagulant therapy warfarin based on genotyping of two genes was described [5]. However, the diagnostic accuracy of individual or pairs of biomarkers is likely to be limited as only a fraction of disease-associated genes is predicted to have a large effect on any specific disease; most disease-associated genes have small effects [6]. Yet, the 


\section{Multiple causes}
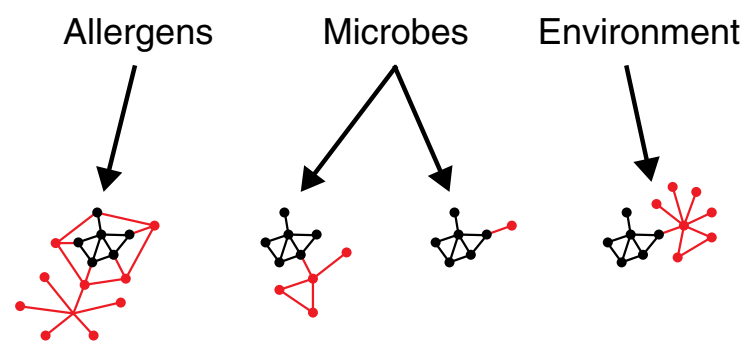

Multiple, overlapping disease mechanisms

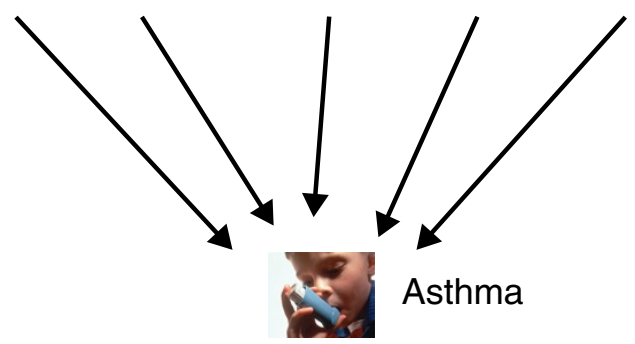

\section{Single clinical phenotype}

Figure $1 \mathrm{~A}$ single disease phenotype can be caused by multiple mechanisms. As an example, asthma can be triggered by allergens, microbes and other environmental factors, each of which may activate different disease mechanisms, which are depicted as shared (black) and specific (red) networks.

combined effect of these small-effect genes may be large. Thus, the accuracy of a biomarker based on a largeeffect gene may vary depending not only on variations in that gene, but also on variations in the many genes with small effects.

Systems medicine is an emerging discipline that aims to address the problem that a disease is rarely caused by malfunction of one individual gene product, but instead depends on multiple gene products that interact in a complex network [7]. Here, we explain how and why systems medicine, and specifically network approaches, can be used to assist clinical decision making and to identify underlying disease mechanisms. We focus on the use of disease modules to uncover pathogenic mechanisms and describe how these can be extended into multilayer networks. We finish by discussing the current problems and limitations of network and systems approaches and suggest possible solutions. We also highlight the necessary steps for clinical implementation. We focus on systems medicine as a network-based approach to analysis of high-throughput and routine clinical data to predict disease mechanisms to diagnoses and treatments.

\section{Systems and network medicine to support clinical decision-making}

Similar to many evolving medical disciplines, there is no generally accepted definition of systems medicine, although different proposals are available $[8,9]$. Some view it as an interdisciplinary approach that integrates research data and clinical practice and others view it as fusion of systems biology and bioinformatics with a focus on disease and the clinic. Recent articles have described systems medicine as a high-precision, mathematical model of variables from different genomic layers that relate to clinical outcomes such as treatment response $[10,11]$. Rather than trying to distinguish between systems medicine and other disciplines, our review is based on the premise that systems medicine is a natural extension of, or is complementary to, current models for clinical decision-making.

In general, clinical decisions are based on a diagnostic model consisting of multilayered pattern recognition of multiple data inputs linked to scientific reasoning about causality. This diagnostic model can be exemplified by pneumonia. On a phenotypic level, pneumonia is often characterized by fever and symptoms or signs of changes in the respiratory tract. This layer of information can be linked to data (such as radiographic imaging, laboratory tests for inflammatory signs of infection and microbial tests) that suggest the cause of the disease. The physician may need to take into account other layers, including socioeconomic and environmental factors. For example, if the patient is homeless and a smoker, this is likely to affect diagnosis, treatment and the innate immune response of the patient to the infection. Thus, in the case of pneumonia, accurate diagnostic decisions can be made by pattern recognition and reasoning.

However, for many diseases, diagnosis is more difficult. The external causes, disease mechanisms or the involvement of cells, tissues or organs may be highly complex or only partially known. In such cases, the physician would be helped by a formal diagnostic model that gave decisional support by presenting the variables so that contributory disease mechanisms can be elucidated and diagnostic predictions computed. One approach is to use a template in which omics clinical variables are organized into a network to understand disease mechanisms and make diagnostic predictions. Such a template would naturally build on the current diagnostic model of pattern recognition. Using this diagnostic model would allow different clinical variables, such as symptoms and laboratory variables, to be described in different network layers. In this way, multilayer network models can be constructed that include all known relevant variables, ranging from genetic variants to environmental factors.

In summary, the potential advantage of a multilayer network model is that it provides a framework in which 
to organize and analyze all relevant disease data simultaneously, thereby informing and improving the decisional pathway of medical professionals and patients [12]. Before we look at how networks and modules can be used to uncover disease mechanisms, we first provide an overview of networks in biology.

\section{A brief introduction to networks}

Networks provide graphical representations of complex systems. In the context of cellular networks, molecules such as genes and proteins are represented as nodes, and the interactions among them as links. In a landmark article in 1999, it was shown that networks in technological, social and biological systems have common designs that are governed by simple and quantifiable organizing principles [13]. Key findings were that a fraction of the nodes serve as hubs with multiple links, whereas the vast majority of nodes have few links. The hubs often have large individual effects, in contrast to the nodes with few links. The hubs contribute to the small world property of networks: all nodes in a network are generally connected by a limited number of links. Another important characteristic is that functionally related nodes tend to be highly interconnected and co-localize in networks, thereby forming modules $[7,14]$ (Table 1).

In the context of disease, disease-associated genes identified by omics studies can be computationally mapped on to models of the human protein-protein interaction (PPI) network. In other words, each diseaseassociated gene is mapped on to its matching protein product. The resulting maps have characteristics that are similar to those found in other types of networks. One of the most important characteristics is that functionally related genes tend to co-localize and form disease modules.

\section{Disease modules for understanding pathogenic mechanisms}

Disease modules can help to organize and prioritize disease-associated genes identified by high-throughput

\section{Table 1 Glossary of terms}

\begin{tabular}{ll}
\hline Term & Description \\
\hline Network & $\begin{array}{l}\text { A graphical representation of a complex system. For } \\
\text { example, in a protein network, proteins are nodes, and } \\
\text { interacting proteins are linked by edges }\end{array}$ \\
$\begin{array}{ll}\text { Disease } \\
\text { module }\end{array}$ & $\begin{array}{l}\text { When mapped onto the protein-protein interaction } \\
\text { network, disease-associated genes tend to co-localize and } \\
\text { form networks of functionally related genes. These networks } \\
\text { are referred to as disease modules }\end{array}$ \\
Multilayer & $\begin{array}{l}\text { A module whose nodes and edges are located across } \\
\text { different layers of disease-relevant information. Such layers } \\
\text { could include transcription factor networks, }\end{array}$ \\
& genetic variants and even environmental factors
\end{tabular}

analyses (Figure 2), as well as to provide an overview of disease mechanisms by performing pathway analyses. Disease modules can also help to identify novel disease genes, biomarkers or therapeutic targets. Remarkably, one landmark study for systems medicine was initiated by researchers without a clinical background, who had studied network design principles in model organisms like yeast cells or worms [15]. In 2007, Pujana et al. [16] described a module relevant to breast cancer, and identified a novel candidate gene, $H M M R$, that was validated by functional and genetic studies. Several module-based studies have been performed in other diseases, including cancer [17-20], neurological [21-23], cardiovascular [24], and inflammatory diseases [25-27]. One of the studies showed how protein interaction modules could be used to predict outcome in breast cancer [20]. In a study of autoimmune diseases, mRNA modules were used to predict disease progression based on functional studies of underlying mechanisms [28]. In 2014, a module-based approach for drug discovery was described in rheumatoid arthritis based on a meta-analysis of genome-wide association studies (GWASs) of 100,000 subjects [29].

Analysis of disease modules exploits the general principles of networks, such as alteration of hub genes being likely to have large effects, while alterations in the many genes with few links will likely correspond to smalleffect genes. Thus, specific therapeutic targeting of a hub gene is more likely to be effective than targeting a gene with few interactions. Indeed, genes targeted by drugs have more interactions than other genes [30], which increases the risk that a drug targeting a specific disease gene may have an off-target effect [31]. An important observation is that nodes that are highly interconnected in a network are likely to be functionally related. Thus, novel candidate genes can be found among the interactors of known disease genes [32].

One recent example of a successful module-based approach was based on the assumption that the genes in a module would be co-regulated by the same set of transcription factors (TFs) that regulate a known disease gene, IL13 [33] (Figure 3). Twenty-five putative IL13regulating TFs were knocked down using short interfering RNA (siRNA), of which seven were found to affect IL13. The knockdowns were repeated for these TFs, followed by mRNA microarrays to detect their downstream targets. This led to the identification of a module of highly interconnected genes. That module contained several genes of known relevance to allergy, such as IFNG, IL12, IL4, IL5, IL13 and their receptors. It also contained novel candidate genes, including S100A4, which was validated as a diagnostic and therapeutic candidate by a combination of functional, mouse and clinical studies. A mouse knock-out model showed that S100A4 had extensive phenotypic, cellular and humoral 
(a)

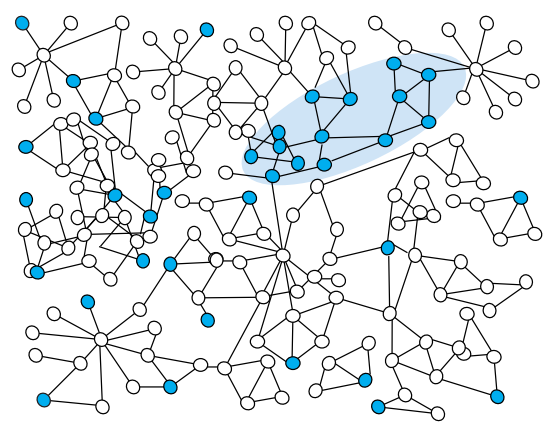

(b)

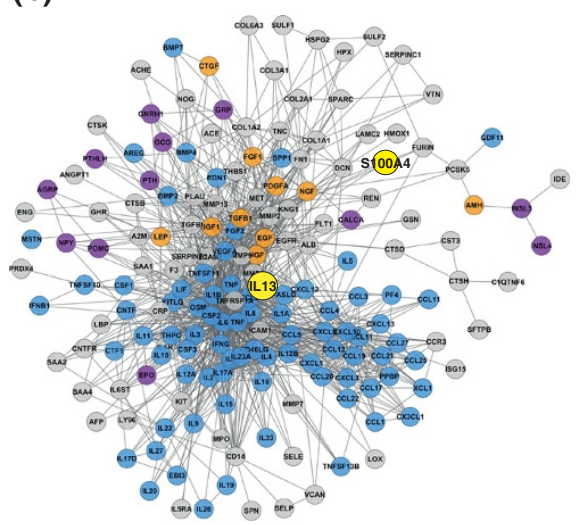

Figure 2 A disease module. (a) Conceptual model of how disease-associated genes (blue nodes), identified by high-throughput analysis, tend to co-localize in the human protein-protein interaction network (white nodes), forming a module (blue oval). The genes in the module are assumed to be more important for the disease than extramodular genes. (b) An actual disease module from allergic patients, showing extracellular proteins that were putatively co-regulated with IL13. Blue nodes are associated with cytokine activity, purple nodes are associated with hormone activity, and orange nodes are associated with growth factor activity according to Gene Ontology Molecular Function. The diagram in (b) is reproduced, with permission, from Bruhn et al. Science Translational Medicine 2014 [33].

effects on allergic inflammation. The therapeutic potential was demonstrated by treatment with a specific antibody, both in the mouse model and in cells from allergic patients.

\section{Multilayer disease modules}

The success of single module approaches in identifying candidate genes prompted researchers to extend it to multiple modules to link genomic, phenotypic and environmental variables together. Rapid development of highthroughput techniques has enabled global analyses of different network layers ranging from DNA to proteins, as well as metabolites and lipids [34,35]. Similar to genes, the variables in each layer can be linked to each other. Consider, for example, one disease module formed by mRNAs and another from single nucleotide polymorphisms (SNPs). If an mRNA and a SNP in each module map to the same protein, they can be linked. This principle can be expanded to all proteins in the module and the overlap tested statistically. Another example is modules formed by genes and their regulators, such as TFs or microRNAs. Genes can be linked if they are regulated by the same microRNAs, and a double-layer module can then be formed by linking microRNAs that regulate the same gene. By combining different highthroughput analyses it is therefore possible to form multilayer disease modules (MLDMs).

Multidimensional models can be used to form rejectable hypotheses of how genes, gene products and regulators interact with each other. For example, does a diseaseassociated SNP in a promoter region of a module gene change the expression of that gene? Does a microRNA regulate its predicted target genes in a module? The clinical relevance of MLDMs lies in that they can provide a framework to identify optimal combinations of diagnostic markers from different layers, based on functional understanding of the pathogenic roles of those markers. For example, microRNAs and genetic variants have been used to examine disease-associated variations in mRNA expression in gliomas, and to predict disease outcome [36,37]. In allergy, functional studies showed that mRNA modules were co-regulated by microRNAs, some of which had hub-like functions and potential diagnostic relevance [38].

An important aspect of MLDMs is that they can be linked to modules formed by other clinical data. For example, a link can be placed between a disease and a gene associated with that disease [39]. Next, diseases that are associated with the same gene can be linked and form a human disease network. The same principle can be applied to the disease genes forming a disease gene network. Such networks are modular and can be linked, so that diseases can be associated with the underlying disease mechanisms. It is also possible to construct and link modules containing other relevant data, such as social and environmental factors (Figure 4). It is of note that the construction of MLDMs is complicated by several technological limitations, which are discussed later in this review.

MLDMs might also be useful for tracking disease over multiple time points. Diseases are dynamic processes rather than static entities, and the underlying processes and time frames may range from hours in rapidly evolving cases, such as meningitis, to decades in cancer. Disease progression is perhaps best understood in cancer. For example, at a molecular level, a study of chronic 


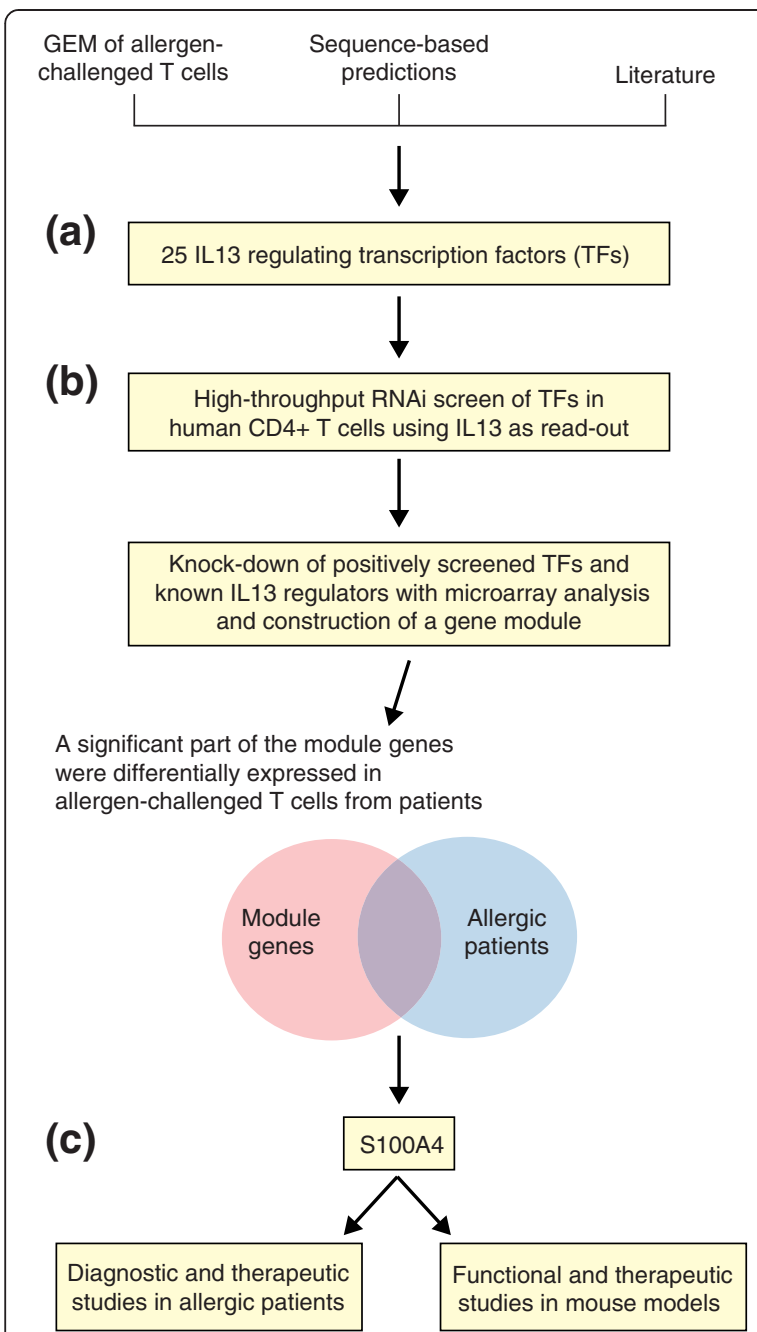

(d)

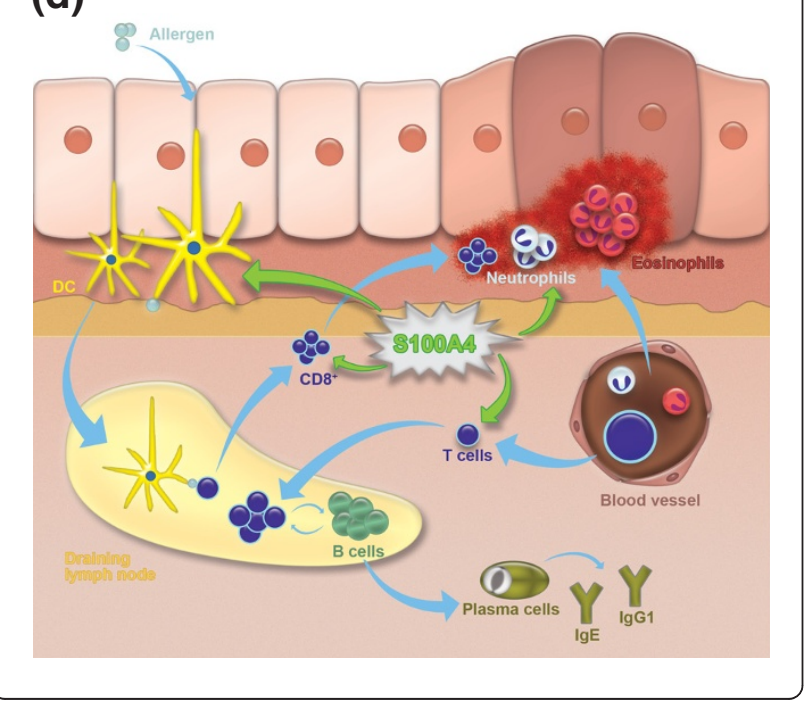

Figure $3 \mathrm{~A}$ module-based approach to identify disease-relevant diagnostic and therapeutic candidate genes in allergy. (a) Twenty-five putative IL13-regulating transcription factors (TFs) were identified by combining data from mRNA microarrays, sequence-based predictions and the literature. (b) IL13-regulating TFs were validated by siRNA-mediated knockdown of the 25 TFs in human total CD4 ${ }^{+} T$ cells polarized toward $T_{H} 2$ using $I L 13$ as a read-out. The target genes of the TFs were identified by combined siRNA knockdown of the positively screened TFs/known IL13-regulating TFs from literature and microarray analyses. This resulted in a module of genes that was co-regulated with $1 / 13$ in $T_{H} 2$-polarized cells and significantly overlapped with differentially expressed genes from allergen-challenged $T$ cells from allergic patients. For further validation experiments, the study focused on module genes that encoded secreted proteins and had not been previously associated with allergy. (c) Functional, diagnostic and therapeutic studies involving one of the module genes, S100A4, were performed in patients with seasonal allergic rhinitis, allergic dermatitis and a mouse model of allergy. (d) Model of S100A4-induced disease mechanisms. Allergic inflammation requires the sensitization of the immune system by allergens, resulting in the production of antigen-specific T cells. The interaction of dendritic cells (DC) in the draining lymph node with $T$ cells is a critical step that is dependent on S100A4. B-cell maturation as a result of T cell-B cell crosstalk (for example, the release of $\mathrm{T}_{H} 2$ cytokines by $T$ cells) leads to the production of $\lg E$ and $\lg G 1$ by plasma cells. Cytokines and chemokines released by $T$ cells stimulate the migration of circulating granulocytes (for example, neutrophils and eosinophils) to the inflammatory site (skin). Differentiation of naive T cells into $\mathrm{CD} 8^{+}$ cytotoxic T cells will exacerbate the skin damage. Blue arrows indicate the flow of the allergic responses. Green arrows indicate the promotion of these processes by S100A4. GEM, gene expression microarray.

lymphocytic leukemia revealed the development of substantial genetic heterogeneity of tumor cells from the same patients over time [40]. Such developments were linked to disease deterioration and variable treatment response. In breast cancer, module kinetics has been directly linked to treatment response; in a subset of patients, treatment with one drug rewired the disease module so that it became sensitive to another drug [41]. Thus, understanding of module kinetics can be exploited for sequential treatment with different drugs. Ideally, this principle should be expanded so that all diseases are staged using MLDMs with omics and routine clinical data integrated. In the future, it may be possible to infer early MLDMs, before patients become symptomatic, allowing preventative medicine.

It is possible that personal MLDMs could become a cornerstone for health care, and could be used for the early diagnosis of changes in module function, based on functional understanding of why disease-causing nodes in the MLDMs change (such as due to a genetic variant). As the bioinformatics principles for analyzing different forms of variables are largely the same, MLDMs could also include other forms of clinical information, such as routine laboratory tests and medical imaging. The versatility and resolution of medical imaging is steadily increasing and is aiming to provide functional understanding of 


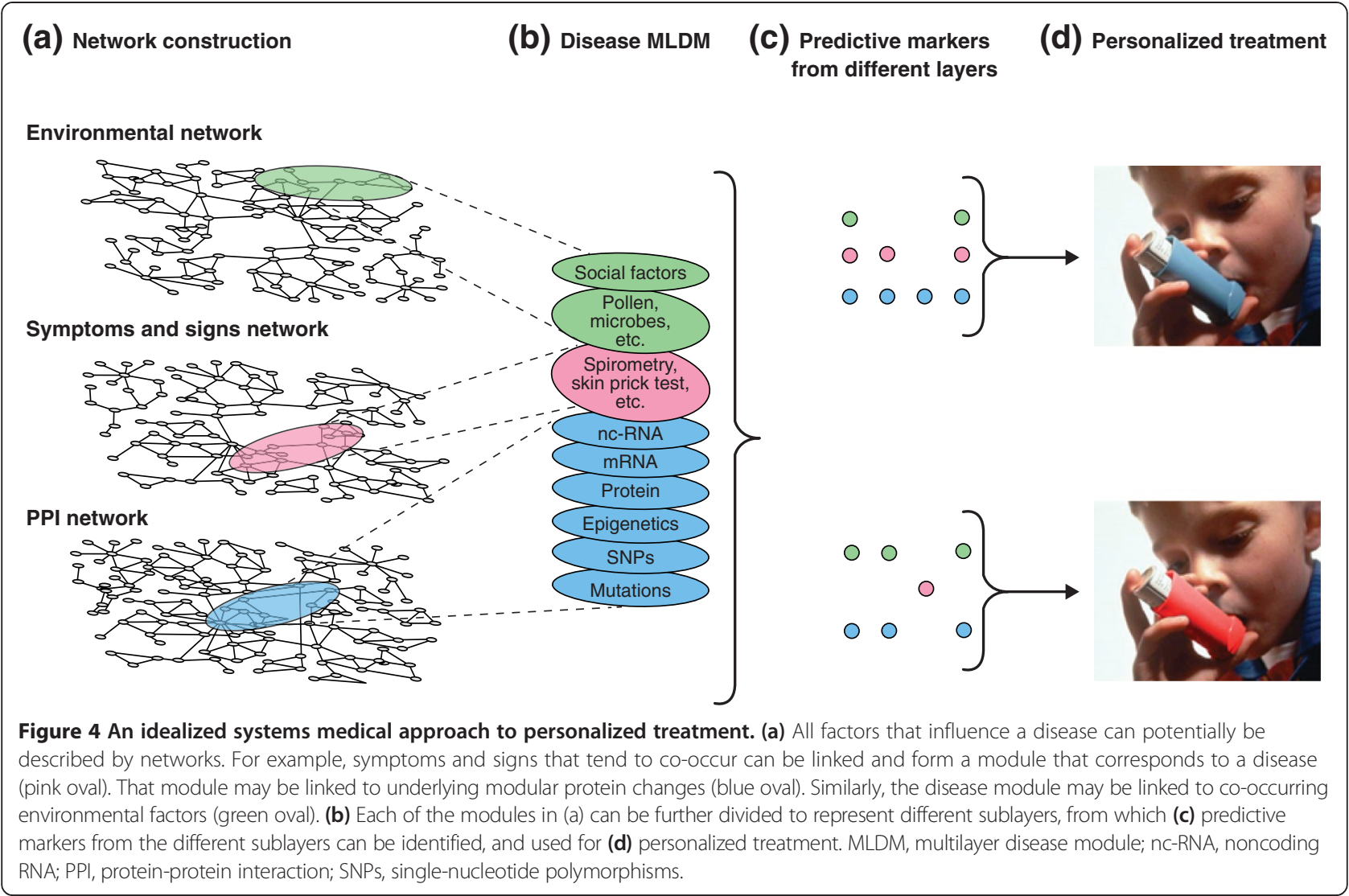

observed structural changes in the human body. This would allow, for example, specific traits imaged in liver cancer to be linked to prognostic gene expression changes [42]. Similarly, obesity traits could be linked to molecular changes [43].

In summary, MLDMs can potentially be used as templates to integrate and analyze multiple layers of diseaserelevant information. Similar to the current diagnostic model discussed above, analyses can be based on functional understanding, but with higher resolution and the option for computational predictions. When the underlying mechanisms are revealed, our view of various common diseases might alter, prompting reclassification of multiple diseases.

\section{Networks to reclassify diseases based on pathogenic mechanisms}

The current diagnostic classification is based on observations of symptoms and signs, associations with external factors (for example, pollen and allergy), and use of diagnostic aids like radiology, and variable molecular knowledge of disease mechanisms. A fundamental problem with this classification system is that the same phenotype may result from multiple disease mechanisms. Thus, if a drug is only effective against one of those mechanisms, its use in patients with different underlying mechanisms will not be therapeutically successful.

Ideally, diagnoses should be based on accurately linking phenotypes with all possible underlying mechanisms. Taking this idea to its extreme would require simultaneously analyzing all possible external causes and mechanisms. Since there is considerable comorbidity, all diseases should also be simultaneously analyzed. Actually, the first steps in this direction have been already taken, using networkbased analyses of public databases and high-throughput data. In a landmark study, Goh et al. [44] mapped human disease genes onto the interactome, and found that genes associated with phenotypically similar diseases tended to co-localize. Similar observations were made for networks derived from expression profiling [45]. This led Barrenas et al. [39] to construct a module-based map of human diseases. Similar to a geographical map, different disease categories should co-localize in different parts of the interactome (Figure 5a). Ideally, such a map could be used as a reference to improve diagnostic accuracy and classification, and better identify diagnostic and therapeutic candidates. However, despite the diseases being very diverse (including metabolic, inflammatory and oncological diseases), they partially overlapped. Thus, instead of being dispersed in the interactome, the disease modules formed a flower-like structure (Figure $5 \mathrm{~b}$ ). The 
(a) Dispersed disease modules

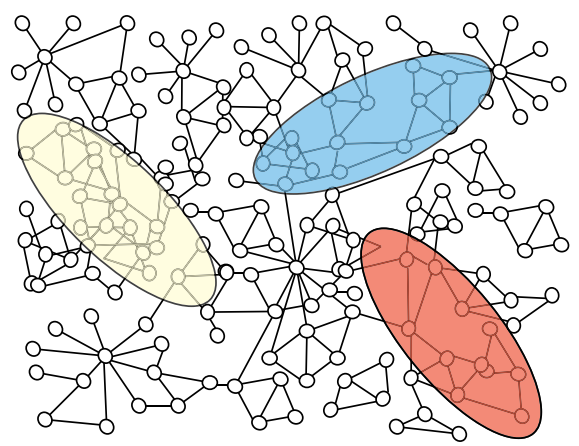

(b) Overlapping disease modules

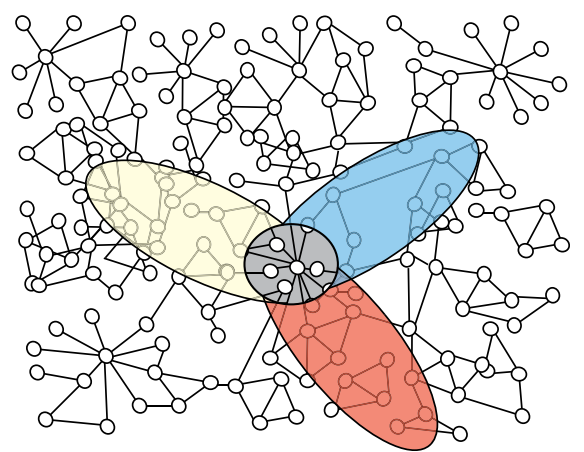

Figure 5 Relationship between different disease modules on the protein-protein interaction network. (a) A hypothetical model of three different diseases mapped on the human protein-protein interaction network. The modules are dispersed in the network. (b) Instead, meta-analysis of mRNA microarray and genome-wide association study data show that disease modules partially overlap and form a shared module (grey) [39]. The shared module has important pathogenic, diagnostic and therapeutic implications.

overlapping disease modules formed a new, shared module with remarkable characteristics. It was enriched for inflammatory, metabolic and proliferative pathways. Since these pathways have key roles in survival, this led to the hypothesis that altered function in one of the pathways may spill over to the others and cause one or more diseases. Indeed, meta-analysis of GWASs representing more than 100 diseases and hundreds of thousands of patients showed that the shared module was highly enriched for SNPs from these diseases [39]. These findings contrast with the dogma that diseases are mainly caused by disease-specific genes, and that nonspecific genes are secondary or irrelevant. Further studies showed that the shared module was more enriched for GWAS genes than disease-specific genes. Moreover, it was highly enriched for known biomarkers and therapeutic targets. Clinical studies showed that the expression profile of the shared module had the potential to stratify allergic patients for treatment with corticosteroids. Because the shared module was highly enriched for GWAS genes it is likely that it has an important causal role, which has diagnostic implications for predictive and preventative medicine $[3,39]$.

Other approaches to disease reclassification have involved mining of electronic health records to search for comorbidity patterns and underlying genetic variants [46-51]. For example, by combining electronic health records and GWASs, Denny et al. [46] showed novel associations. For example, the presence of polymorphisms in IRF4 was linked to skin cancer and actinic keratosis [46]. There are also enormous resources of biomedical relevance available in the public domain that can be analyzed with network-based principles. For example, Medline contains some 20 million abstracts, the Gene Expression Omnibus one million expression experiments, and the Encyclopedia of DNA Elements (ENCODE) more than
2,500 high-throughput experiments. In one study, new indications for known drugs were predicted based on integration of public expression data of more than 100 diseases and expression data from the drugs. For example, an antiulcer drug, cimetidine, was shown to be a therapeutic candidate in lung cancer [52]. In another study, a hypothesis about T-cell differentiation was tested completely in silico, by mining and modeling data in the public domain. All abstracts in MedLine were mined to construct a module relevant for T-cell differentiation. This module was tested by simulated activation and knockdown of individual module genes. The simulation yielded unexpected results, which were validated by analyses of correlation patterns in public mRNA microarray data from different T-cell-associated diseases [53]. It is likely that networkbased analysis of highly diverse data sets with increasingly powerful computational tools will contribute to a new disease taxonomy. Already, there are examples of this, such as in severe asthma [54].

\section{Problems, limitations and opportunities}

Every step of a systems medicine study, including the use of network and module approaches, involves problems and limitations. One problem is that highthroughput analyses often require large sample sizes to obtain statistically significant results, and sufficient samples may be difficult to obtain. In some diseases, it is difficult or impossible to obtain relevant clinical samples, such as neurodegenerative diseases. One solution to this problem, at this stage, may be to focus on particularly tractable diseases. As an example, in seasonal allergic rhinitis, the key external trigger (pollen) and the key cell type (lymphocytes) are both known and readily accessible. The disease occurs at a known time point each year. Thus, it is possible to mimic the disease process by in vitro challenge of $\mathrm{T}$ cells from 
patients outside of the pollen season. It is also possible to perform functional studies of candidate genes in activated $\mathrm{T}$ cells, or in a well-defined mouse model of allergy. The disease process and diagnostic markers can be analyzed locally in the affected organ [33].

Another issue is that many different cell types are often involved in one disease, and more than one may be important. The involvement of multiple cell types in the development of a disease introduces an additional challenge to the generation of meaningful MLDMs from omics data relying on cell mixtures. This may be addressed in the near future by the application of single-cell analysis technologies. Recent developments in sequencing allow determination of single-cell genomes and transcriptomes $[55,56]$, while mass cytometry enables the targeted quantification of proteins and their modifications in different cells from a heterogeneous population [57].

Other challenges arise from technical problems, which include variation in the accuracy and sensitivity of highthroughput techniques. This is particularly so for global protein profiling, which is complex and difficult to perform in a clinical setting. The occurrence in a proteome of various post-translational modifications, SNPs and alternative splicing of proteins further complicates such analyses. However, recent technological advances indicate that targeted proteomics may partly address these limitations and render the analysis of predetermined sets of proteins over large numbers of samples [58,59]. Targeted protein assays may also enable the quantification of highly homologous protein sequences, such as splice variants, protein isoforms and mutated versions of a protein [60], in a clinical laboratory setting. Another emerging targeted proteomic application is the generation of perpetually reusable digitalized maps of the proteomic signals of a sample [61]. The thus generated maps can then be mined using targeted data extraction strategies to quantify disease-related proteins of interest over large cohorts of patient samples. Literature knowledge and MLDM layers that are more easily measured than proteins, such as mRNA or genomic information, could help to identify proteins for such targeted analyses [62]. Similarly, recent technical advances may help to include targeted metabolites and lipids in the MLDMs [63,64].

The bioinformatics analyses involve several problems of their own. For example, important limitations of PPI networks are that they are generally not cell specific, and are constructed based on heterogeneous sources such as literature and databases, experimental data, inferences from high-throughput studies, or computational predictions [65].

A key remaining problem is how to validate results from analyses involving thousands of genes or gene products.
Systems medicine is based on combining genome-scale validation strategies with detailed studies of individual factors. Therefore, it is mandatory to follow recommendations for multiscale analysis [66], thereby strictly limiting false discovery [67]. Recently, these analyses have been anchored to MLDMs, by providing stepwise criteria for the use of omics-based predictors in clinical trials [68].

On a genomic scale, an important validation principle is to test for genomic concordance. In other words, to test if there is concordance between different layers in an MLDM. For example, it is possible to validate by examining if disease modules that are derived from mRNA microarray analyses are enriched for SNPs identified by independent GWASs of the same diseases. Another form of genome-scale validation is to examine if siRNA-mediated knockdowns of predicted upstream genes in a module result in altered expression of downstream module genes. If these two genome-scale analyses support the findings, then detailed functional and clinical studies can be performed, including mouse disease models [33].

\section{Clinical implementation of systems and network medicine}

There are already examples of gene testing being used in the clinic. Diagnostic products to stratify breast cancer based on gene expression profiling are commercially available, such as the MammaPrint [69]. MLDMs could also be used to stratify patients for individualized medicine based on functional understanding of why patients do or do not respond to a particular drug. This could, in turn, lead to development of novel drugs for nonresponders, directed against mechanisms not targeted by existing drugs. MLDMs could also be used for repositioning of drugs that have not reached the market because of low efficacy or side effects.

The clinical implementation of systems medicine would require extensive clinical, administrative and educational adaptations. One current problem is that very few clinicians are involved in systems medical research, education or implementation. Yet, systems medicine is beginning to become a part of the curricula of many medical schools (for example, http://gumc.georgetown. edu/spi/systemsmedicine).

The European Commission has launched a project aiming to draw up a road map for the clinical implementation of systems medicine (https://www.casym.eu). This road map is based on integrating the views from different relevant stakeholders, including clinicians, basic researchers, representatives of the pharmaceutical industry, funding bodies and government health agencies. Educational programs for the training of health professionals at different stages of their careers, starting from medical school, have already started in the USA and some European countries. 
It is important to recognize that systems medical principles are in line with clinical reasoning, and perhaps can be seen as a natural extension that permits formalized reasoning about pathogenic mechanisms, as well as diagnostic predictions.

\section{Conclusions and future directions}

Many of the main challenges facing modern health care arise from the complex and heterogeneous characteristics of common diseases. The same phenotype may result from different mechanisms, and each mechanism will require a different treatment. Ideally as many phenotypes, genes and other disease-associated variables as possible should be studied together in order to reclassify diseases based on functional understanding of underlying mechanisms. However, this involves multiple, large-scale methodological, economical and ethical challenges, which are only partially resolved.

The rapid development of systems medicine is illustrated by a prospective study of 100 healthy subjects, known as the Hundred Person Wellness Project, which was started in March 2014. Blood, urine and stool samples will be regularly analyzed for multiple biomarkers or microbes, and participants will wear digital devices that monitor physical activity, sleep patterns and blood pressure. The aim is to predict and prevent disease. If successful, the study will expand to include 100,000 subjects [70].

The study suggests that the predictive and personalized medicine based on MLDMs will become a reality. From an idealized perspective, a global description of MLDMs for all diseases and relevant cell types would lead to increased understanding of the relationships between pathogenic mechanisms and disease phenotypes. This would include understanding of comorbidity and subgroups. An important clinical use would be diagnostic reclassification of diseases, which in turn could contribute to more effective diagnosis, drug development and treatment. The next natural aim would be to include a time axis in the reclassified diagnostic disease map. In such a map, diseases should be staged by defining MLDMs at different time points. Ideally, such staging should extend to early and even presymptomatic stages. If so, this could help to identify markers that aid in the prediction and perhaps prevention of disease before it becomes symptomatic. The identification of early and presymptomatic MLDMs based on clinical data would be a very large undertaking that would require population-based studies where the subjects are followed for several years. Alternatively, it could be possible to infer early MLDMs based on analyses of animal models of diseases or in human cells exposed to known external disease triggers, such as $\mathrm{T}$ cells exposed to allergen. The clinical advantages of predictive and preventative medicine can be exemplified by early treatment of rheumatoid arthritis and multiple sclerosis, which reduces the risk of debilitating disease [71]. If these examples can be generalized, medicine would be likely to change from reactive to proactive.

Clinical research is rapidly entering the era of low-cost personalized omics, and we believe that systems medicine is ideally placed to make sense of this sea of complex data, resulting in tangible improvements in patient care and treatment.

\section{Abbreviations}

GWAS: Genome-wide association study; MLDM: Multilayer disease module; PPI: Protein-protein interaction; SNP: Single-nucleotide polymorphism; siRNA: Short interfering RNA; TF: Transcription factor.

\section{Competing interests}

The authors declare that they have no competing interests.

\section{Acknowledgements}

We thank Robert Liljenström, Jan Ernerudh, Antonella Muraro and Stefan Thor for valuable comments and discussion. This work has been supported by the European Commission under the Seventh Framework Programme, CASyM, the Swedish Medical Research Council, and strategic funding from Linköping University.

\section{Author details}

${ }^{1}$ Centre for Individualized Medicine, Department of Pediatrics, Faculty of Medicine, 58185 Linköping, Sweden. ${ }^{2}$ Department of Physics, Biology and Computer Science, Center for Complex Network Research, Northeastern University, Boston, MA 02115, USA. ${ }^{3}$ Department of Neurology, University of California, San Francisco, CA 94143, USA. ${ }^{4}$ Center for Biological Sequence Analysis, Department of Systems Biology, Technical University of Denmark, DK-2800 Lyngby, Denmark. ${ }^{5}$ Novo Nordisk Foundation Center for Protein Research, Faculty of Health Sciences, University of Copenhagen, DK-2200 Copenhagen, Denmark. ${ }^{6}$ Airways Disease Section, National Heart and Lung Institute, Imperial College London, London SW3 6LY, UK. 'Department of Neurology and Neuroscience, Georgetown University Medical Center, Washington, DC 20057, USA. ${ }^{8}$ European Molecular Biology Laboratory, 69117 Heidelberg, Germany. ${ }^{9}$ MRC Human Genetics Unit, MRC IGMM, University of Edinburgh, Edinburgh EH4 2XU, UK. ${ }^{10}$ Institute of Biochemistry, University of Zürich, 8093 Zürich, Switzerland. ${ }^{11}$ Catalan Institute of Oncology, Bellvitge Biomedical Research Institute (IDIBELL), Barcelona 08908, Spain. ${ }^{12}$ Systems Biology of Gene Regulatory Elements, Max-Delbrück-Center for Molecular Medicine, Robert-Rössle-Strasse 10, 13125 Berlin, Germany. ${ }^{13}$ Cambridge Institute for Medical Research, University of Cambridge, Cambridge Biomedical Campus, Cambridge CB2 OXY, UK. ${ }^{14}$ Department of Medicine, University of Cambridge School of Clinical Medicine, Addenbrooke's Hospital, Cambridge CB2 0QQ, UK. ${ }^{15}$ Department of Respiratory Medicine, Academic Medical Centre, University of Amsterdam, 1100 DE Amsterdam, The Netherlands. ${ }^{16}$ Center of Neuroimmunology and Department of Neurology, Institut d'investigacions Biomèdiques August Pi i Sunyer (IDIBAPS), Hospital Clinic of Barcelona, 08028 Barcelona, Spain.

\section{Published online: 17 October 2014}

\section{References}

1. What happened to personalized medicine? Nat Biotechnol 2012, 30:1

2. Hood $L$, Flores M: A personal view on systems medicine and the emergence of proactive $\mathrm{P} 4$ medicine: predictive, preventive, personalized and participatory. New Biotechnol 2012, 29:613-624.

3. Gustafsson M, Edström M, Gawel D, Nestor CE, Wang H, Zhang H, Barrenäs F, Tojo J, Kockum I, Olsson T, Serra-Musach J, Bonifaci N, Pujana MA, Ernerudh J, Benson M: Integrated genomic and prospective clinical studies show the importance of modular pleiotropy for disease susceptibility, diagnosis and treatment. Genome Med 2014, 6:17.

4. McCarthy JJ, McLeod HL, Ginsburg GS: Genomic medicine: a decade of successes, challenges, and opportunities. Sci Transl Med 2013, 5:189sr184 
5. Pirmohamed $M$, Burnside $G$, Eriksson $N$, Jorgensen $A L$, Toh $C H$, Nicholson $T$, Kesteven P, Christersson C, Wahlstrom B, Stafberg C, Zhang JE, Leathart JB, Kohnke H, Maitland-van der Zee AH, Williamson PR, Daly AK, Avery P, Kamali F, Wadelius M, EU-PACT Group: A randomized trial of genotype-guided dosing of warfarin. N Engl J Med 2013, 369:2294-2303.

6. Petretto E, Liu ET, Aitman TJ: A gene harvest revealing the archeology and complexity of human disease. Nat Genet 2007, 39:1299-1301.

7. Barabasi AL, Gulbahce N, Loscalzo J: Network medicine: a network-based approach to human disease. Nat Rev Genet 2011, 12:56-68.

8. Zhang H, Gustafsson M, Nestor C, Chung FK, Benson M: Targeted omics and systems medicine: personalising care. Lancet Respir Med 2014, 14:70188-70182.

9. O'Shea P: Future medicine shaped by an interdisciplinary new biology. Lancet 2012, 379:1544-1550.

10. Kolch W, Kholodenko BN: Systems medicine: opportunities and challenges for systems biology approaches. FEBS J 2013, 280:5937.

11. Wolkenhauer O, Auffray C, Brass O, Clairambault J, Deutsch A, Drasdo D, Gervasio F, Preziosi L, Maini P, Marciniak-Czochra A, Kossow C, Kuepfer L, Rateitschak K, Ramis-Conde I, Ribba B, Schuppert A, Smallwood R, Stamatakos $\mathrm{G}$, Winter $\mathrm{F}$, Byrne $\mathrm{H}$ : Enabling multiscale modeling in systems medicine. Genome Med 2014, 6:21.

12. Sun X, Vilar S, Tatonetti NP: High-throughput methods for combinatorial drug discovery. Sci Transl Med 2013, 5:205rv201.

13. Barabasi AL, Albert R: Emergence of scaling in random networks. Science 1999, 286:509-512.

14. Mitra K, Carvunis AR, Ramesh SK, Ideker T: Integrative approaches for finding modular structure in biological networks. Nat Rev Genet 2013, 14:719-732.

15. Jeong $H$, Mason SP, Barabasi AL, Oltvai ZN: Lethality and centrality in protein networks. Nature 2001, 411:41-42.

16. Pujana MA, Han JD, Starita LM, Stevens KN, Tewari M, Ahn JS, Rennert G, Moreno V, Kirchhoff T, Gold B, Assmann V, Elshamy WM, Rual JF, Levine D, Rozek LS, Gelman RS, Gunsalus KC, Greenberg RA, Sobhian B, Bertin N, Venkatesan K, Ayivi-Guedehoussou N, Solé X, Hernández P, Lázaro C, Nathanson KL, Weber BL, Cusick ME, Hill DE, Offit K, et al: Network modeling links breast cancer susceptibility and centrosome dysfunction. Nat Genet 2007, 39:1338-1349.

17. Chuang HY, Lee E, Liu YT, Lee D, Ideker T: Network-based classification of breast cancer metastasis. Mol Syst Biol 2007, 3:140.

18. Wuchty S, Zhang A, Walling J, Ahn S, Li A, Quezado M, Oberholtzer C, Zenklusen JC, Fine HA: Gene pathways and subnetworks distinguish between major glioma subtypes and elucidate potential underlying biology. J Biomed Inform 2010, 43:945-952.

19. Ergun A, Lawrence CA, Kohanski MA, Brennan TA, Collins JJ: A network biology approach to prostate cancer. Mol Syst Biol 2007, 3:82.

20. Taylor IW, Linding R, Warde-Farley D, Liu Y, Pesquita C, Faria D, Bull S, Pawson T, Morris Q, Wrana JL: Dynamic modularity in protein interaction networks predicts breast cancer outcome. Nat Biotechnol 2009, 27:199-204

21. Ray M, Ruan J, Zhang W: Variations in the transcriptome of Alzheimer's disease reveal molecular networks involved in cardiovascular diseases. Genome Biol 2008, 9:R148.

22. Hwang D, Lee IY, Yoo H, Gehlenborg N, Cho JH, Petritis B, Baxter D, Pitstick R, Young R, Spicer D, Price ND, Hohmann JG, Dearmond SJ, Carlson GA, Hood LE: A systems approach to prion disease. Mol Syst Biol 2009, 5:252.

23. Villoslada P, Steinman L, Baranzini SE: Systems biology and its application to the understanding of neurological diseases. Ann Neurol 2009, 65:124-139

24. Presson AP, Sobel EM, Papp JC, Suarez CJ, Whistler T, Rajeevan MS, Vernon SD, Horvath S: Integrated weighted gene co-expression network analysis with an application to chronic fatigue syndrome. BMC Syst Biol 2008, 2:95.

25. Calvano SE, Xiao W, Richards DR, Felciano RM, Baker HV, Cho RJ, Chen RO, Brownstein BH, Cobb JP, Tschoeke SK, Miller-Graziano C, Moldawer LL, Mindrinos MN, Davis RW, Tompkins RG, Lowry SF, Inflamm and Host Response to Injury Large Scale Collaboration Research Program: A network-based analysis of systemic inflammation in humans. Nature 2005, 437:1032-1037.

26. Liu M, Liberzon A, Kong SW, Lai WR, Park PJ, Kohane IS, Kasif S: Network-based analysis of affected biological processes in type 2 diabetes models. PLOS Genet 2007, 3:e96.

27. Hägg S, Skogsberg J, Lundström J, Noori P, Nilsson R, Zhong H, Maleki S, Shang MM, Brinne B, Bradshaw M, Bajic VB, Samnegård A, Silveira A, Kaplan LM, Gigante B, Leander K, de Faire U, Rosfors S, Lockowandt U, Liska J,
Konrad P, Takolander R, Franco-Cereceda A, Schadt EE, Ivert T, Hamsten A, Tegnér J, Björkegren J: Multi-organ expression profiling uncovers a gene module in coronary artery disease involving transendothelial migration of leukocytes and LIM domain binding 2: the Stockholm Atherosclerosis Gene Expression (STAGE) study. PLoS Genet 2009, 5:e1000754.

28. McKinney EF, Lyons PA, Carr EJ, Hollis JL, Jayne DR, Willcocks LC, Koukoulaki M, Brazma A, Jovanovic V, Kemeny DM, Pollard AJ, Macary PA, Chaudhry AN, Smith KG: A CD8+ T cell transcription signature predicts prognosis in autoimmune disease. Nat Med 2010, 16:586-591.

29. Okada Y, Wu D, Trynka G, Raj T, Terao C, Ikari K, Kochi Y, Ohmura K, Suzuki A, Yoshida S, Graham RR, Manoharan A, Ortmann W, Bhangale T, Denny JC, Carroll RJ, Eyler AE, Greenberg JD, Kremer JM, Pappas DA, Jiang L, Yin J, Ye L, Su DF, Yang J, Xie G, Keystone E, Westra HJ, Esko T, Metspalu A, et al: Genetics of rheumatoid arthritis contributes to biology and drug discovery. Nature 2014, 506:376-381.

30. Yildirim MA, Goh Kl, Cusick ME, Barabasi AL, Vidal M: Drug-target network Nat Biotechnol 2007, 25:1119-1126.

31. Kuhn M, Al Banchaabouchi M, Campillos M, Jensen LJ, Gross C, Gavin AC, Bork P: Systematic identification of proteins that elicit drug side effects. Mol Syst Biol 2013, 9:663.

32. Lim J, Hao T, Shaw C, Patel AJ, Szabo G, Rual JF, Fisk CJ, Li N, Smolyar A, Hill DE, Barabási AL, Vidal M, Zoghbi HY: A protein-protein interaction network for human inherited ataxias and disorders of Purkinje cell degeneration. Cell 2006, 125:801-814

33. Bruhn S, Fang Y, Barrenäs F, Gustafsson M, Zhang $H$, Konstantinell A, Krönke A, Sönnichsen B, Bresnick A, Dulyaninova N, Wang H, Zhao Y, Klingelhöfer J, Ambartsumian N, Beck MK, Nestor C, Bona E, Xiang Z, Benson M: A generally applicable translational strategy identifies S100A4 as a candidate gene in allergy. Sci Trans/ Med 2014, 6:218ra4.

34. McShane LM, Cavenagh MM, Lively TG, Eberhard DA, Bigbee WL, Williams PM Mesirov JP, Polley MY, Kim KY, Tricoli JV, Taylor JM, Shuman DJ, Simon RM, Doroshow $\mathrm{JH}$, Conley BA: Criteria for the use of omics-based predictors in clinical trials. Nature 2013, 502:317-320

35. Wheelock CE, Goss VM, Balgoma D, Nicholas B, Brandsma J, Skipp PJ, Snowden S, Burg D, D'Amico A, Horvath I, Chaiboonchoe A, Ahmed H, Ballereau S, Rossios C, Chung KF, Montuschi P, Fowler SJ, Adcock IM, Postle AD, Dahlén SE, Rowe A, Sterk PJ, Auffray C, Djukanovic R, U-BIOPRED Study Group: Application of'omics technologies to biomarker discovery in inflammatory lung diseases. Eur Resp J 2013, 42:802-825.

36. Jörnsten R, Abenius T, Kling T, Schmidt L, Johansson E, Nordling TE, Nordlander B, Sander C, Gennemark P, Funa K, Nilsson B, Lindahl L, Nelander S: Network modeling of the transcriptional effects of copy number aberrations in glioblastoma. Mol Syst Biol 2011, 7:486.

37. Genovese G, Ergun A, Shukla SA, Campos B, Hanna J, Ghosh P, Quayle SN, Rai K, Colla S, Ying H, Wu CJ, Sarkar S, Xiao Y, Zhang J, Zhang H, Kwong L, Dunn K, Wiedemeyer WR, Brennan C, Zheng H, Rimm DL, Collins JJ, Chin L: microRNA regulatory network inference identifies miR-34a as a novel regulator of TGF-beta signaling in glioblastoma. Cancer Discov 2012, 2:736-749.

38. Chavali S, Bruhn S, Tiemann K, Saetrom P, Barrenas F, Saito T, Kanduri K, Wang $\mathrm{H}$, Benson $\mathrm{M}$ : MicroRNAs act complementarily to regulate disease-related mRNA modules in human diseases. RNA 2013 19:1552-1562.

39. Barrenas F, Chavali S, Alves AC, Coin L, Jarvelin MR, Jornsten R, Langston MA, Ramasamy A, Rogers G, Wang H, Benson M: Highly interconnected genes in disease-specific networks are enriched for disease-associated polymorphisms. Genome Biol 2012, 13:R46.

40. Landau DA, Carter SL, Stojanov P, McKenna A, Stevenson K, Lawrence MS, Sougnez C, Stewart C, Sivachenko A, Wang L, Wan Y, Zhang W, Shukla SA, Vartanov A, Fernandes SM, Saksena G, Cibulskis K, Tesar B, Gabriel S, Hacohen N, Meyerson M, Lander ES, Neuberg D, Brown JR, Getz G, Wu CJ: Evolution and impact of subclonal mutations in chronic lymphocytic leukemia. Cell 2013, 152:714-726

41. Lee MJ, Ye AS, Gardino AK, Heijink AM, Sorger PK, MacBeath G, Yaffe MB: Sequential application of anticancer drugs enhances cell death by rewiring apoptotic signaling networks. Cell 2012, 149:780-794.

42. Segal E, Sirlin CB, Ooi C, Adler AS, Gollub J, Chen X, Chan BK, Matcuk GR, Barry $C T$, Chang HY, Kuo MD: Decoding global gene expression programs in liver cancer by noninvasive imaging. Nat Biotechnol 2007, 25:675-680.

43. Lidell ME, Betz MJ, Dahlqvist Leinhard O, Heglind M, Elander L, Slawik M, Mussack T, Nilsson D, Romu T, Nuutila P, Virtanen KA, Beuschlein F, Persson 
A, Borga $M$, Enerbäck S: Evidence for two types of brown adipose tissue in humans. Nat Med 2013, 19:631-634.

44. Goh Kl, Cusick ME, Valle D, Childs B, Vidal M, Barabasi AL: The human disease network. Proc Natl Acad Sci U S A 2007, 104:8685-8690.

45. Zhernakova A, Withoff S, Wijmenga C: Clinical implications of shared genetics and pathogenesis in autoimmune diseases. Nat Rev Endocrinol 2013, 9:646-659

46. Denny JC, Bastarache L, Ritchie MD, Carroll RJ, Zink R, Mosley JD, Field JR, Pulley JM, Ramirez AH, Bowton E, Basford MA, Carrell DS, Peissig PL, Kho AN, Pacheco JA, Rasmussen LV, Crosslin DR, Crane PK, Pathak J, Bielinski SJ, Pendergrass SA, Xu H, Hindorff LA, Li R, Manolio TA, Chute CG, Chisholm RL, Larson EB, Jarvik GP, Brilliant MH, et al: Systematic comparison of phenome-wide association study of electronic medical record data and genome-wide association study data. Nat Biotechnol 2013, 31:1102-1111.

47. Rzhetsky A, Wajngurt D, Park N, Zheng T: Probing genetic overlap among complex human phenotypes. Proc Natl Acad Sci U S A 2007, 104:1 1694-11699.

48. Blair DR, Lyttle CS, Mortensen JM, Bearden CF, Jensen AB, Khiabanian H, Melamed R, Rabadan R, Bernstam EV, Brunak S, Jensen LJ, Nicolae D, Shah $\mathrm{NH}$, Grossman RL, Cox NJ, White KP, Rzhetsky A: A nondegenerate code of deleterious variants in Mendelian loci contributes to complex disease risk. Cell 2013, 155:70-80.

49. Zitnik M, Janjic V, Larminie C, Zupan B, Przulj N: Discovering disease-disease associations by fusing systems-level molecular data. Sci Rep 2013, 3:3202

50. Jensen $P B$, Jensen $\perp$, Brunak S: Mining electronic health records: towards better research applications and clinical care. Nat Rev Genet 2012, 13:395-405.

51. Hidalgo CA, Blumm N, Barabasi AL, Christakis NA: A dynamic network approach for the study of human phenotypes. PLoS Comp Biol 2009, 5:e1000353.

52. Sirota M, Dudley JT, Kim J, Chiang AP, Morgan AA, Sweet-Cordero A, Sage J, Butte AJ: Discovery and preclinical validation of drug indications using compendia of public gene expression data. Sci Transl Med 2011, 3:96ra77.

53. Pedicini M, Barrenas F, Clancy T, Castiglione F, Hovig E, Kanduri K, Santoni D, Benson M: Combining network modeling and gene expression microarray analysis to explore the dynamics of Th1 and Th2 cell regulation. PLoS Comp Biol 2010, 6:e1001032.

54. Auffray C, Adcock IM, Chung KF, Djukanovic R, Pison C, Sterk PJ: An integrative systems biology approach to understanding pulmonary diseases. Chest 2010, 137:1410-1416.

55. Navin N, Kendall J, Troge J, Andrews P, Rodgers L, McIndoo J, Cook K, Stepansky A, Levy D, Esposito D, Muthuswamy L, Krasnitz A, McCombie WR, Hicks J, Wigler M: Tumour evolution inferred by single-cell sequencing. Nature 2011, 472:90-94.

56. Tang F, Barbacioru C, Wang Y, Nordman E, Lee C, Xu N, Wang X, Bodeau J, Tuch BB, Siddiqui A, Lao K, Surani MA: mRNA-Seq whole-transcriptome analysis of a single cell. Nat Methods 2009, 6:377-382.

57. Bodenmiller B, Zunder ER, Finck R, Chen TJ, Savig ES, Bruggner RV, Simonds EF, Bendall SC, Sachs K, Krutzik PO, Nolan GP: Multiplexed mass cytometry profiling of cellular states perturbed by small-molecule regulators. Nat Biotechnol 2012, 30:858-867.

58. Picotti P, Clément-Ziza M, Lam H, Campbell DS, Schmidt A, Deutsch EW, Röst H, Sun Z, Rinner O, Reiter L, Shen Q, Michaelson JJ, Frei A, Alberti S, Kusebauch U, Wollscheid B, Moritz RL, Beyer A, Aebersold R: A complete mass-spectrometric map of the yeast proteome applied to quantitative trait analysis. Nature 2013, 494:266-270.

59. Rifai N, Gillette MA, Carr SA: Protein biomarker discovery and validation: the long and uncertain path to clinical utility. Nat Biotechnol 2006, 24:971-983.

60. Picotti P, Aebersold R: Selected reaction monitoring-based proteomics: workflows, potential, pitfalls and future directions. Nat Methods 2012, 9:555-566.

61. Liu Y, Huttenhain R, Collins B, Aebersold R: Mass spectrometric protein maps for biomarker discovery and clinical research. Expert Rev Mol Diagn 2013, 13:811-825.

62. Hüttenhain R, Soste M, Selevsek N, Röst H, Sethi A, Carapito C, Farrah T, Deutsch EW, Kusebauch U, Moritz RL, Niméus-Malmström E, Rinner O, Aebersold R: Reproducible quantification of cancer-associated proteins in body fluids using targeted proteomics. Sci Trans/ Med 2012, 4:142ra94.

63. Dumas ME, Kinross J, Nicholson JK: Metabolic phenotyping and systems biology approaches to understanding metabolic syndrome and fatty liver disease. Gastroenterology 2014, 146:46-62.
64. Saliba AE, Vonkova I, Ceschia S, Findlay GM, Maeda K, Tischer C, Deghou S, van Noort V, Bork P, Pawson T, Ellenberg J, Gavin AC: A quantitative liposome microarray to systematically characterize protein-lipid interactions. Nat Methods 2014, 11:47-50.

65. Schadt EE, Bjorkegren JL: NEW: network-enabled wisdom in biology, medicine, and health care. Sci Trans/ Med 2012, 4:115rv111.

66. Sung J, Wang Y, Chandrasekaran S, Witten DM, Price ND: Molecular signatures from omics data: from chaos to consensus. Biotechnol J 2012, 7:946-957.

67. Broadhurst DI, Kell DB: Statistical strategies for avoiding false discoveries in metabolomics and related experiments. Metabolomics 2006, 2:171-196.

68. McShane LM, Cavenagh MM, Lively TG, Eberhard DA, Bigbee WL, Williams PM, Mesirov JP, Polley MYC, Kim KY, Tricoli JV, Taylor JM, Shuman DJ, Simon RM, Doroshow JH, Conley BA: Criteria for the use of omics-based predictors in clinical trials. Nature 2013, 502:317-320.

69. Kittaneh M, Montero AJ, Gluck S: Molecular profiling for breast cancer: a comprehensive review. Biomark Cancer 2013, 5:61-70.

70. Gibbs WW: Medicine gets up close and personal. Nature 2014, 506:144-145.

71. Hauser SL, Chan JR, Oksenberg JR: Multiple sclerosis: prospects and promise. Ann Neurol 2013, 74:317-327.

\section{doi:10.1186/s13073-014-0082-6}

Cite this article as: Gustafsson et al:: Modules, networks and systems medicine for understanding disease and aiding diagnosis. Genome Medicine 2014 6:82. 